\title{
Cryptography Based Light-Weight Attribute Encryption Scheme for Internet of Things
}

\author{
Dhruva M S \\ Dept. of Computer Science and \\ Engineering, Malnad College of \\ Engineering, Hassan, India.
}

\author{
Gururaj H L \\ Dept. of Computer Science and \\ Engineering, Malnad College of \\ Engineering, Hassan, India.
}

\author{
Ramesh B \\ Dept. of Computer Science and \\ Engineering, Malnad College of \\ Engineering, Hassan, India.
}

\begin{abstract}
For those organization for vast number about IoT devices, progressively vast amounts about information need aid produced, which abandons provision suppliers confronting new tests especially for administration to security. Information security might make attained utilizing cryptographic encryption strategies. Cryptographic keys are needed will unscramble data, Furthermore must a chance to be disseminated to An specific best approach will commissioned information clients that perform dissimilar errands with respect to information. To deal with an extensive amount about units generating trickles for data, an adaptable and fine-grained get control result thought to a chance to be utilized. A guaranteeing approach need as of late rose In view of Attribute-Based encryption (ABE) that gives adaptable What's more fine-grained get control. This framework employments quality based encryption to IOT gadgets. Those different clients previously, an association will oblige to entry different information produced Eventually Tom's perusing diverse sensors. Hence, in this system, best the clients who need aid commissioned will right specific information camwood right it. The way used to scramble that information is itself encrypted Also sent should client. When a commissioned client solicitation those data, as much genuineness is main checked and the qualities of the asked information are sent on as much email. He may be at that point obliged with enters this data. These qualities would use to unscramble those encrypted enter. Those decrypted fact that thus used to unscramble the information
\end{abstract}

Keywords - Internet of things; security; attribute-based encryption; pre-computation; sensors.

\section{INTRODUCTION}

Cryptographic keys are required should unscramble information and are dispersed to sanctioned client that perform unique errands for information. Those methodology that is utilized to the framework will be quality based encryption. Quality built encryption (ABE) characterizes right control approaches In view of different qualities connected with information clients. There would A large number sorts for $\mathrm{ABE}$ the place Ciphertext arrangement ABE will be utilized within the framework the place the coin content is connected with a security approach and client unscrambling keys are connected with a situated of qualities. The qualities connected with mystery key must fulfill those security arrangements. Cryptographic keys need aid obliged with unscramble data, Furthermore must be conveyed clinched alongside specific route should sanctioned information clients that perform dissimilar errands with respect to information. Will wrist bindings an expansive amount from claiming units generating trickles from claiming data, an adaptable What's more finegrained right control result if make utilized. A guaranteeing approach need as of late risen dependent upon Attribute-Based encryption (ABE) that gives adaptable Furthermore finegrained entry control. ABE understands those attribute-based entry control model that characterizes right control arrangements In light of separate.

Qualities connected with Realities users, location, or information fundamentals. $\mathrm{ABE}$ doesn't define a whole endorse system, at it give graceful strategy will cryptographically force attribute-based confirmation run approaches.

To occasion, in the all over IoT motivation for example, advanced mobile city, information will be Typically assembled starting with distinctive wellsprings possessed by transformed official domains (e. G. , advanced mobile phones, Also state funded alternately private moving providers). Those information aggregation might a chance to be crazy of the user's information Furthermore information transmit might a chance to be previously, plaintext. Since the gigantic together information is shared "around Dissimilar to departments, which might be right by illicit clients on foundation genuine exertion or Indeed make used to mischief those holders of the information if no security cutoff is constructed on it. An additional illustration is a unit IoT application, which is physical condition or therapeutic observing. Usually, the information gathered Toward the constitution sensors connected to an elderly representative alternately tolerant ought to make interminably sent of the lilac wine waiter of the checkup focus or healing center What's more close-by best by the particular doctors, since the form information need aid know delicate information. Those protection might be broken on it will be transmitted to plaintext alternately there will be no suitableness get oversee aggravated once it, which might prompt grave outcome. Previously, adding, those multi-hop remote show importance mode On IoT may be also powerless will eavesdropping.

Information security might a chance to be getting toward property about cryptographic encryption systems. Cryptographic keys need aid necessary to unscramble data, also 
must be conveyed clinched alongside a perceiving route with permitted information clients that perform unique errands on information. To deal with an expansive amount from claiming units generating trickles for data, an adaptable Furthermore fine-grained entry control result ought a chance to be utilized. A guaranteeing methodology need as of late rose In view of Attribute-Based encryption (ABE) that gives adaptable What's more fine-grained get control. ABE understands the attributebased right control model that characterizes confirmation control approaches In light of different qualities connected with information users, environment, alternately information components. ABE doesn't sake a whole commission system, Anyhow it gives a rich technique with cryptographically authorize attribute-based get control approaches. A standout amongst the significant profits of $\mathrm{ABE}$ is that it doesn't require sending those information clinched alongside a secure channel, or storing it Previously, An trusted stockpiling site. Then afterward ABE encryption Eventually Tom's perusing those information source, information could just be decrypted Eventually Tom's perusing clients with those fancied qualities.

Attribute-based encryption (ABE) is a glass house from claiming identity-based encryption [7] which permits clients with scramble and unscramble messages In view of qualities What's more contact structures. Ciphertext-policy attributebased encryption (CP-ABE) is a sort from claiming $\mathrm{ABE}$ schemes the place the unscrambling fact that connected with a user's quality situated.

The encryptor characterizes those get structure to ensure delicate information such-and-such best clients whose qualities fulfill those entry structure might unscramble the messages. Because of this decent property, CP-ABE need pulled in a considerable measure from claiming consideration (e. G. [8][10]) to requisitions for example, entry control. Large portions CP-ABE scheme (e. G. [11]- [19]) have been recommended for Different purposes for example, such that short ciphertext Furthermore full security evidences. though, we establish no $\mathrm{CP}-\mathrm{ABE}$ plan with expressive right structure in the writing tending to the span issue of unscrambling keys, which appears to be on a chance to be a detriment because of asset utilization. Every last bit existing $\mathrm{CP}-\mathrm{ABE}$ schemes fair from the issue for long unscrambling keys, over which the period may be subject to the number from claiming qualities. This issue gets all the more obvious, the point when $\mathrm{CP}-\mathrm{ABE}$ unscrambling keys would connect on storage-constrained units. Due to those Notoriety for lightweight units What's more of service requisitions about $\mathrm{CP}-\mathrm{ABE}$, in this employment, we advocate An provably secure $\mathrm{CP}-\mathrm{ABE}$ table so as to offer short unscrambling keys, which need aid relevant for enter stockpiling over lightweight gadgets.

\section{LITERATURE SURVEY}

An arrangement for understanding perplexing gets control for encrypted information that we bring Ciphertext-Policy Attribute-Based encryption. By utilizing our systems encrypted information might be held private regardless of those capacity server will be untrusted[1].Those existing ABE schemes are dependent upon exorbitant bilinear pairing, which make them not suitableness to those resources-constraint IoT requisitions.
A lightweight no-pairing $\mathrm{ABE}$ plan In view of elliptic bend cryptography (ECC) will be recommended to deliver those security and protection issues in IoT[2].

Information protection camwood a chance to be attained utilizing cryptographic encryption strategies. Cryptographic keys need aid required to unscramble data, What's more must make conveyed over a specific manner with sanctioned information clients that perform unique errands on information. With wrist bindings an expansive amount from claiming units generating trickles about data, an adaptable and fine-grained entry control result ought to a chance to be used [1].

Nouha Oualha, Kim Thuat Nguyen Since those ciphertext will be assembled with those get control policy, those information wellspring need full control through who could unscramble the information. [1].

Leveraging on the favorable circumstances advertised Eventually Tom's perusing the CP-ABE, a few extensions (e.g., [6]) have been produced to apply those plan in the connection from claiming resource-constrained IoT gadgets. The recommended results don't gatherings give another $\mathrm{CP}-\mathrm{ABE}$ cryptographic development for every se, However augment those unique plan. A few of the suggested results point should relieve the issue from claiming asset limitations, by relying on the accessibility of internet semi trusted proxies in the system that perform the exorbitant cryptographic pick the right structure choose who might right the message. The thought for $\mathrm{CP}-\mathrm{ABE}$ might have been principal suggested Eventually Tom's perusing.

We recommend a ciphertext-policy attribute-based encryption clinched alongside which the right structures are and entryways [13], [16].

Unscrambling magic connected with a trait situated a camwood unscramble ciphertxts with the entry structure $p$ when $\mathrm{p} \perp$ An. Basically significant, the unscrambling fact that constant-size What's more free of the number of qualities. That's only the tip of the iceberg precisely, those unscrambling fact that created for two assembly components best and the extent could be 672 odds at mainly beneath 80 -bit security prerequisite. Those recommended $\mathrm{CP}-\mathrm{ABE}$ plan will be provably secure in the specific security model.

\section{PROPOSED WORK}

The framework comprises for key server, IoT units Also cloud server. IoT sends sensor information of the enter server for encryption. Magic server.

1) Encrypts those information from claiming sensors sent by IoT gadgets Furthermore saves it to cloud server.

2) Generates quality to sensor id al-adha.

Client registers for interesting client sake, international ID Furthermore machine name which will be saved in the way server. Magic server In light of machine name, client name and international ID generates An 32 touch magic utilizing SHA algorithm which will be put away in the key server. Following the long haul at the logs in the way server authenticates the 
client utilizing those 32 touch magic produced formerly throughout enrollment. Once the client may be verified An rundown for sensors (e.g. light, temperature, Humidity) is shown and client could select from those rundown Also ask for to the information of the cloud server. Cloud server sends the sensor id al-adha of the magic server Furthermore solicitations for its qualities. Enter server generates the qualities on the support about sensor id al-adha utilizing CPA(Cipher quick approach algorithm) Also sends it of the cloud server. Cloud server ahead getting the attributes, sends those qualities alongside encrypted information of the sensor of the client. Utilizing these qualities those client decrypts those sensor information.

Attribute-based encryption (ABE) might have been To begin with present by Sahai Also Waters done [20]. There would two variant for ABE: Key-Policy ABE What's more Ciphertext-Policy ABE [11].

- KP-ABE: clinched alongside An KP-ABE proposal, those ciphertext encrypting a message will be associated with a set about qualities. An unscrambling magic issued Eventually Tom's perusing a power is connected with a right structure. Those ciphertext could a chance to be decrypted for those unscrambling way On Furthermore just if those trademark set about ciphertext fulfills the right structure from claiming unscrambling magic.

- $\mathrm{CP}-\mathrm{ABE}$ : to a CP-ABE scheme, on the contrary, the ciphertext encrypts a message with a right structure same time an unscrambling fact that connected with situated about qualities. The unscrambling condition will be similar: whether and just if that quality situated fulfills those right structure.

In [11] yet they didn't delicate any develop [12]. Not long after from that point onward, Bethencourt, Sahai and Waters [12] proposed the principal $\mathrm{CP}-\mathrm{ABE}$ origination. At that point, Cheung and Newport [13] arranged another CP-ABE in which the get to structures are AND doors. CP-ABE close steady size ciphertexts have been proposed. Herranz et al. [17] and Chen et al. [22] projected CP-ABE conspire with consistent size ciphertexts under the limit get to structure. Zhou and Huang [16] proposed a CP-ABE plot with consistent size ciphertexts under AND doors get to structure. CP-ABE plans with consistent size ciphertexts are additionally contemplated in [24] and [25].

A ciphertext-arrangement quality based encryption plan is quiet of four calculations: Setup, Encrypt, KeyGen, and Decrypt.

P - > An expansive prime, the limited field with $p$ components is indicated by F_p.

E-> An elliptic bend over the limited field F_p, which has a subgroup of expansive prime request $\mathrm{q}$.

q-> An expansive prime, which is utilized to indicate the request of $\mathrm{G}$ in $\mathrm{E}$ over $\mathrm{F} \_p$.

$\mathrm{Zq}->\mathrm{A}$ limited number field, whose components set is $\{0$, $1, \ldots, q-1\}$.

$$
\mathrm{Zq} *->\mathrm{Zq} * \mathrm{Zq}-\{0\}
$$

$\mathrm{G}->\mathrm{A}$ construct point in light of the elliptic bend $\mathrm{E}$.

$\mathrm{GE}->A$ subgroup of $E$ with the request of $q$.

$\mathrm{O}$ - > The zero component of an elliptic bend assemble.

HMAC(M, IK) - > A cryptographic hash capacity to produce the hash-based message confirmation code for $\mathrm{M}$ as indicated by the trustworthiness key IK.

$\mathrm{H}(\mathrm{M})$ - > A hash work.

MK-> The ace private key of the ABE plot.

PK - > The ace open key of the ABE plot.

Params - > The open key parameters of the ABE conspire.

ENC(M, EK) - >A symmetric encryption calculation, which scramble the message $M$ with the key EK.

DEC(C, EK)- > A symmetric unscrambling calculation, which decode the figure content $\mathrm{C}$ with the key EK.

$\mathrm{k}$ - >The number of the credits used to encode information.

$\mathrm{n}->$ The number of the characteristics in a framework.

PS-> One point scalar augmentation.

- Setup method: Taking as info a security parameter $\lambda$ and a universe of characteristics $\{\mathrm{A} 1, \mathrm{~A} 2, \ldots, \mathrm{An}\}$, the setup calculation yields open parameter MPK and an ace mystery key MSK.

- Encryption: winning as info a get to structure P, open parameters MPK and a message M, the encryption calculation Enc $[\mathrm{P}, \mathrm{M}]$ yields a ciphertext $\mathrm{C}$.

- KeyGeneration: Taking as info a list of capabilities An, open parameters MPK and the ace undercover key MSK, the key development calculation yields the decoding key of A, which is signify by skA.

- Decryption: Taking as info a ciphertext $\mathrm{C}$ created with get to arrangement $\mathrm{P}$, open parameters MPK and the unscrambling key skA comparing to the quality set $\mathrm{A}$, the decoding calculation Dec[C, P, skA, A] yields the message $\mathrm{M}$ or yields $\perp$.

\section{CONCLUSION}

This paper portrays a pre-calculation system connected to the CP-ABE encryption calculation permitting overcoming the computational expenses of encryption that scale with the manysided quality of the get to arrangement and the quantity of properties. The proposed system can be considered as an advancement of the encryption calculation to relieve the down to earth issues in executing $\mathrm{CP}-\mathrm{ABE}$ on asset compelled gadgets. We have exhibited the vitality sparing additions that are accomplished by this system, as far as calculation expenses. On the off chance that the capacity prerequisite of the strategy turns into a vital concern, a half breed approach exchanging pre-calculations and on-request calculations can be formulated to conquer this issue. 


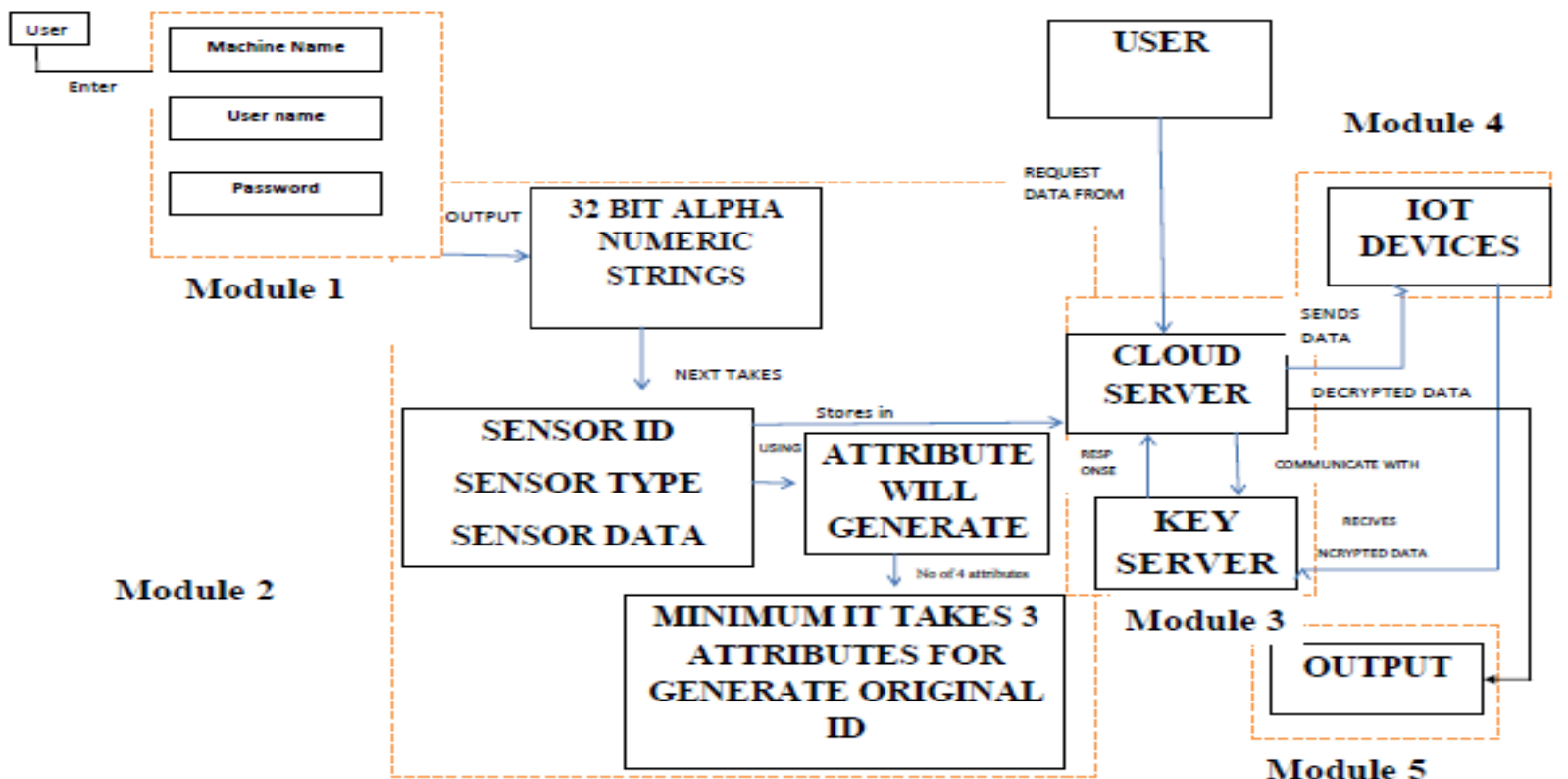

Figure 1. Proposed work

\section{RESUlTS AND ANALYSIS}

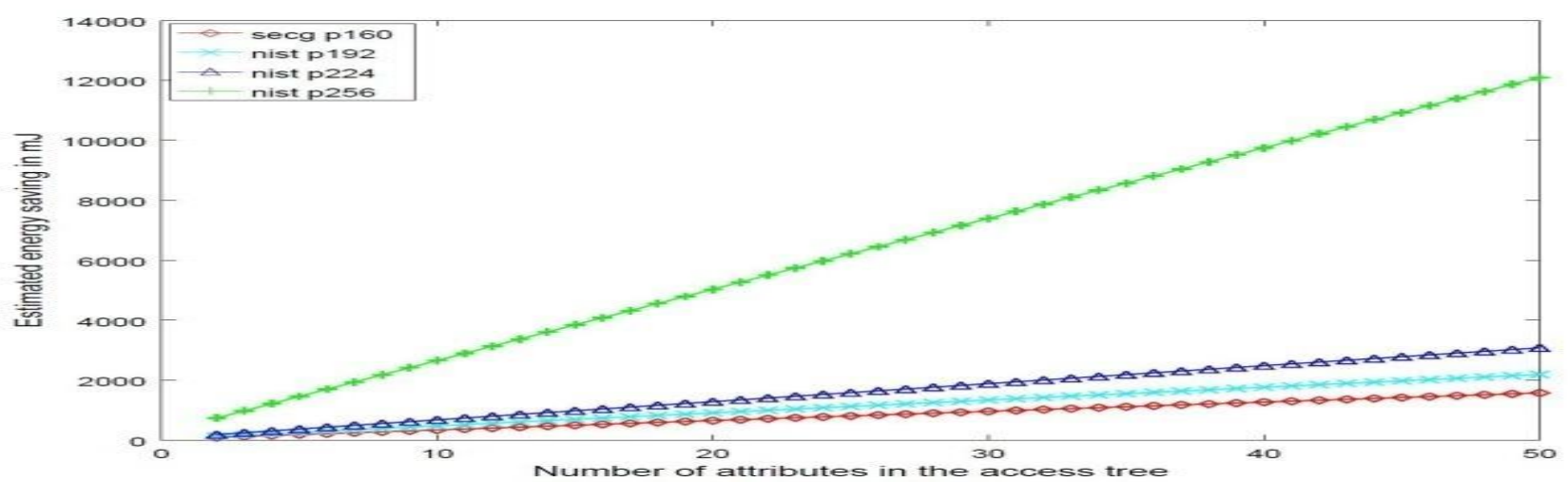

Figure 2. Encryption Process

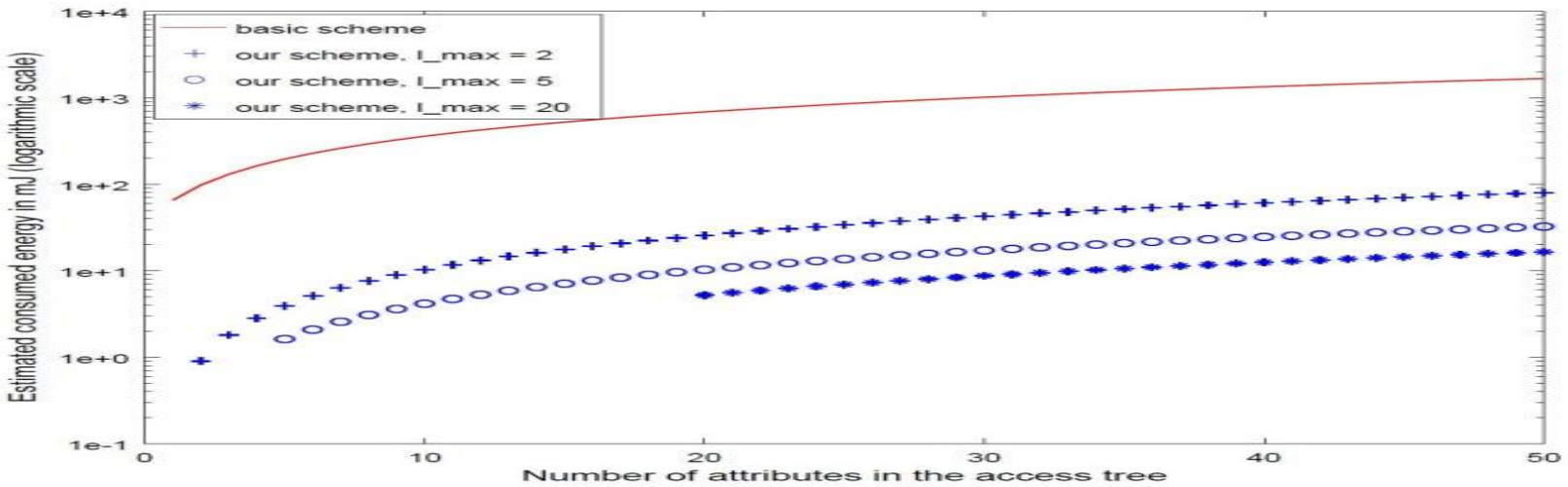

Figure 3. Decryption Process 


\section{REFERENCES}

[1] S. Vaudenay, "On privacy models for RFID," in Proc. ASIACRYPT, 2007, vol. 4833, pp. 68-87.

[2] C. Delerablée, "Identity-based broadcast encryption with constant size ciphertexts and private keys," in Proc. attribute-based encryption: Achieving full security through selective techniques," in Proc. CRYPTO, 2012, vol. 7417, pp. 180-198.

[3] F. Guo, Y. Mu, and W. Susilo, "Identity-based traitor tracing with short private key and short ciphertext," in Proc. ESORICS, 2012, vol. 7459, pp. 609-626.

[4] F. Guo, Y. Mu, and Z. Chen, "Identity-based encryption: How to decrypt multiple ciphertexts using a single decryption key," in Proc. Pairing, 2007, vol. 4575, pp. 392-406.

[5] F. Guo, Y. Mu, Z. Chen, and L. Xu, "Multi-identity single-key decryption without random oracles," in Proc. Inscrypt, 2007, vol. 4990, pp. 384-398.

[6] H. Guo, C. Xu, Z. Li, Y. Yao, and Y. Mu, "Efficient and dynamic key management for multiple identities in identity-based systems," Inf. Sci., vol. 221, pp. 579-590, Feb. 2013.

[7] D. Boneh and M. K. Franklin, "Identity-based encryption from the weil pairing," in Proc. CRYPTO, 2001, vol. 2139, pp. 213-229.

[8] G. Wang, Q. Liu, and J. Wu, "Hierarchical attribute-based encryption for fine-grained access control in cloud storage services," in Proc. ACM Conf. Comput. Commun. Security, 2010, pp. 735-737.

[9] J. Hur and D. K. Noh, "Attribute-based access control with efficient revocation in data outsourcing systems," IEEE Trans. Parallel Distrib. Syst., vol. 22, no. 7, pp. 1214-1221, Jul. 2011.

[10] Z. Wan, J. Liu, and R. H. Deng, "Hasbe: A hierarchical attributebased solution for flexible and scalable access control in cloud computing," IEEE Trans. Inf. Forensics Security, vol. 7, no. 2, pp. 743-754, Apr. 2012.

[11] V. Goyal, O. Pandey, A. Sahai, and B. Waters, "Attribute-based encryption for fine-grained access control of encrypted data," in Proc. ACM Conf. Comput. Commun. Security, 2006, pp. 89-98.

[12] J. Bethencourt, A. Sahai, and B. Waters, "Ciphertext-policy attributebased encryption," in Proc. IEEE Symp. Security Privacy, May 2007, pp. 321-334.

[13] L. Cheung and C. C. Newport, "Provably secure ciphertext policy abe," in Proc. ACM Conf. Comput. Commun. Security, 2007, pp. 456-465.

[14] B. Waters, "Ciphertext-policy attribute-based encryption: An expressive, efficient, and provably secure realization," in Proc. Public Key Cryptograph., 2011, vol. 6571, pp. 53-70.

[15] K. Emura, A. Miyaji, A. Nomura, K. Omote, and M. Soshi, "A ciphertext-policy attribute-based encryption scheme with constant ciphertext length," in Proc. ISPEC, 2009, vol. 5451, pp. 13-23.

[16] Z. Zhou and D. Huang, "On efficient ciphertext-policy attribute based encryption and broadcast encryption: Extended abstract," in Proc. ACM Conf. Comput. Commun. Security, 2010, pp. 753-755.

[17] J. Herranz, F. Laguillaumie, and C. Ràfols, "Constant size ciphertexts in threshold attribute-based encryption," in Proc. Public Key Cryptography, 2010 , vol. 6056, pp. 19-34.
[18] A. B. Lewko, T. Okamoto, A. Sahai, K. Takashima, and B. Waters, "Fully secure functional encryption: Attribute-based encryption and (hierarchical) inner product encryption," in Proc. EUROCRYPT, 2010, vol. 6110, pp. 62-91.

[19] A. B. Lewko and B. Waters, "New proof methods for . attribute- based encryption:achieving full security through selective techniques", in proc.crypto,2012 vol.7417, pp.180-198.

[20] A. Sahai and B. Waters, "Fuzzy identity-based encryption," in Proc. EUROCRYPT, 2005, vol. 3494, pp. 457-473

Dhruva M.S. completed his B.E. degree in computer science and engineering from Visvesvaraya Technology University, Belgaum, India .Pursuing M.Tech degree in computer science and engineering from Visvesvaraya Technological University, Belgaum. Currently he is working as Lecturer in the Department of Computer Science and Engineering at Rajeev Institute of Technology, Hassan, India. His areas of interest include multimedia networks, Compiler Design and Algorithm.

Gururaj H.L. completed his B.E. and M.Tech degrees in computer science and engineering from Visvesvaraya Technological University, Belgaum, India in 2009 and 2013 respectively. Currently he is working as an assistant professor in the Department of Computer Science and Engineering at Malnad College of Engineering, Hassan, India. His areas of interest include congestion control algorithms, security issues in cloud computing and routing protocols for multi-hop wireless networks.

Ramesh B. completed his B.E. degree in computer science and engineering from Mysore University, Karnataka, India in 1991 and M.Tech degree in computer science from DAVV, Indore, Madhya Pradesh, India, in 1995 and Ph.D. degree from Anna University in 2009. Currently he is working as a professor and the head in the Department of Computer Science and Engineering at Malnad College of Engineering, Hassan, India. His current research interests lie in the areas of congestion control QoS-aware routing algorithms in ad hoc networks and multimedia networks
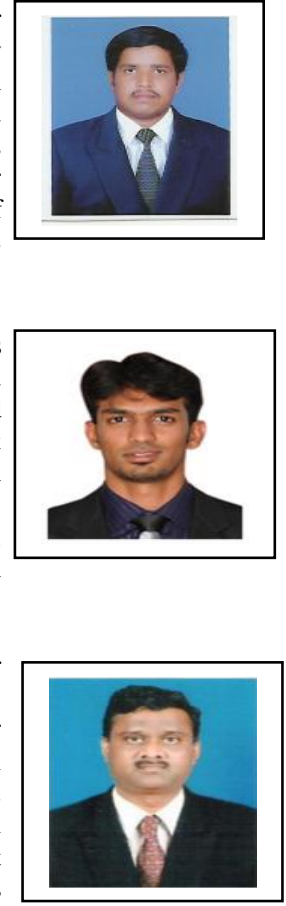

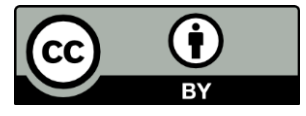

(C) 2017 by the author(s); licensee Empirical Research Press Ltd. United Kingdom. This is an open access article distributed under the terms and conditions of the Creative Commons by Attribution (CC-BY) license. (http://creativecommons.org/licenses/by/4.0/). 